\title{
Elapsed Time Between Breaths
}

National Cancer Institute

\section{Source}

National Cancer Institute. Elapsed Time Between Breaths. NCI Thesaurus. Code

C158355.

The amount of time between one breath to the next breath calculated by inspiration time plus expiration time. 